\title{
On joint synchronization of clock offset and skew for Wireless Sensor Networks under exponential delay
}

\author{
Mei Leng and Yik-Chung Wu
}

\begin{abstract}
In this paper, the problem of clock synchronization for Wireless Sensor Network (WSN) under exponential delay is analyzed based on two-way message exchange mechanism. The Maximum Likelihood Estimator (MLE) for joint estimation of the clock offset and clock skew is derived, and an approximate Cramer-Rao Lower Bound (CRLB) is also developed. Simulation results verify that the proposed estimator gives improved performance compared to an existing algorithm.
\end{abstract}

\section{INTRODUCTION}

Wireless Sensor Network (WSN), emerged as an important research area in recent years, consists of many smallscale miniature devices (or sensor nodes) capable of onboard sensing, computing and communications. WSNs are used in industrial and commercial applications to monitor data that would be difficult or inconvenient to monitor using wired equipment, such as monitoring the health status of environment, controlling industrial machines and home appliances, fire detection and object tracking, etc. [1] [2]. Most of these applications require collaborative execution of a distributed task amongst a large set of synchronized sensor nodes. Furthermore, data fusion, power management and transmission scheduling require all the nodes running on a common time frame. However, every individual sensor in a WSN has its own clock. Different clocks will drift from each other with time due to many factors, such as imperfection of the oscillators and environmental changes. This makes clock synchronization between different nodes an indispensable piece of infrastructure.

Clock synchronization is not an easy task in practice due to several unique properties of WSN. The first and most important one is the limited power supply in low-end sensor nodes. Due to harsh operating conditions, nodes in WSNs are mostly left unattended for their lifetimes without any maintenance or battery replacement. To save power, each synchronization procedure should be simple and the frequency of re-synchronization should be low. This makes simplicity and accuracy the primary concerns of clock synchronization algorithms for WSNs.

The second challenge of clock synchronization in WSN is the unknown message delays in physical and MAC layers. Kopetz and Ochsenreiter [4] for the first time analyzed the process of message delay and decompose the unknown delay

\footnotetext{
The authors are with the Department of Electrical and Electronic Engineering, The University of Hong Kong, Hong Kong. Email: \{meileng, ycwu\}@eee.hku.hk.

The work was supported in part by the HKU Seed Funding Programme, Project No. 200611159233
}

into several components: send time, access time, transmission time, propagation time, reception time and receive time. These delay components can be grouped into two portions: the fixed delay and the random delay. The fixed delay is usually unknown, and if it is not modeled explicitly, it will be treated as a part of time offset, thus lowering the accuracy of timing parameter estimation. On the other hand, the random delay has been modeled as random variables following different distributions (such as Gaussian distribution, exponential distribution, Gamma and Weibull distribution) in the literature based on different justifications and applications, and the difficulty of designing an optimal algorithm for joint estimation of clock offset and clock skew largely depends on the modeling of this random delay.

When the random delay follows Gaussian distribution, the optimal estimator has been given in [7]. However, as pointed out in [3], in many cases, (e.g., when the point-to-point HRX (Hypothetical Reference Connection) topology is of interest), the link delay between two nodes is appropriately represented as a regular M/M/1 queue, and the random delay should be modeled as exponential random variables. In this case, it is much more difficult to design the optimal clock synchronizer. Jeske [5] derived the Maximum Likelihood Estimator (MLE) of clock offset with an unknown fixed delay. But unfortunately the clock skew is not considered, which may result in frequent re-synchronization. Therefore, Noh et al. [6] proposed an algorithm for joint estimation of clock offset and clock skew by treating the fixed delay as a nuisance paramter (denoted as EMLLE in this paper). Unfortunately, in EMLLE, not all the available data are used, thus the performance of the EMLLE is limited.

In this paper, the MLE for joint estimation of the clock offset and clock skew is derived when the fixed delay is unknown and the random delay follows exponential distribution. In general, the Cramer-Rao Lower Bound (CRLB) does not exist for exponential delay, but by using a mild approximation, an approximate CRLB is derived. Simulation results confirm that the proposed estimator provides improved performance over the EMLLE.

\section{SYSTEM MODEL}

We consider the synchronization between a parent node $P$ and its child node $S$ based on a two-way timing message exchange mechanism as shown in Fig. 1. In the $i^{\text {th }}$ round of message exchange, node $S$ sends a synchronization message to 


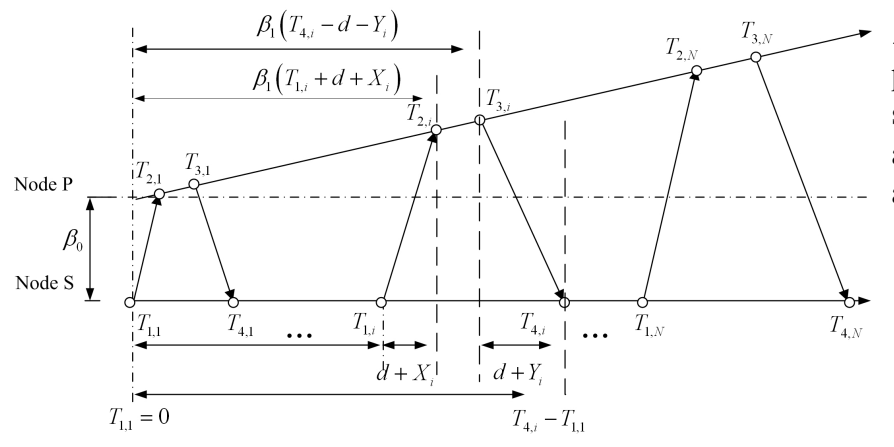

Fig. 1. Two-way time-stamps exchange between two nodes $S$ and $P$.

node $P$ at $T_{1, i}$. Node $P$ records its time $T_{2, i}$ at the reception of that message, and replies node $S$ at $T_{3, i}$. The replied message contains both time-stamps $T_{2, i}$ and $T_{3, i}$. Then node $S$ records the reception time of node $P$ 's reply as $T_{4, i}$. Note that $T_{1, i}$ and $T_{4, i}$ are the time stamps recorded by the clock of node $S$, while $T_{2, i}$ and $T_{3, i}$ are recorded by that of node $P$. After $N$ rounds of message exchange, node $S$ obtains a set of time stamps $\left\{T_{1, i}, T_{2, i}, T_{3, i}, T_{4, i}\right\}_{i=1}^{N}$. The above procedure can be modeled as [6]

$$
\begin{aligned}
& T_{2, i}=\beta_{1} \times T_{1, i}+\beta_{0}+\beta_{1} \times\left(d+X_{i}\right), \\
& T_{3, i}=\beta_{1} \times T_{4, i}+\beta_{0}-\beta_{1} \times\left(d+Y_{i}\right),
\end{aligned}
$$

where $\beta_{0}$ and $\beta_{1}$ represents the clock offset and clock skew of node $S$ with respect to node $P$, respectively; $d$ stands for the fixed portion of message delay from one node to another; and $X_{i}$ and $Y_{i}$ are variable portions of the message delay. Based on the reasons explained in Section I and the fact that the down/up links between two nodes are usually symmetric, $X_{i}$ and $Y_{i}$ are assumed to be independent and identical distributed (i.i.d.) and follow exponential distribution with common rate parameter. The goal is to estimate clock offset $\beta_{0}$ and clock skew $\beta_{1}$ based on the observation of a set of time-stamps $\left\{T_{1, i}, T_{2, i}, T_{3, i}, T_{4, i}\right\}_{i=1}^{N}$.

\section{MAXIMUM LIKELIHOOd ESTIMATOR (MLE) FOR CLOCK OFFSET AND SKEW}

First notice that the fixed delay $d$ is unknown and it is a nuisance parameter in the case of clock synchronization. By observing that the uplink and downlink undergo the same amount of fixed delay, we can rewrite the original model by adding (1) to (2), and we have

$$
T_{2, i}+T_{3, i}=\beta_{1} \times\left(T_{1, i}+T_{4, i}\right)+2 \beta_{0}+\beta_{1} \times\left(X_{i}-Y_{i}\right)
$$

Dividing the above equation by $\beta_{1}$, defining $\theta_{1}=1 / \beta_{1}$ and $\theta_{0}=\beta_{0} / \beta_{1}$, and stacking all the time-stamps in matrix form, the model becomes

$\left[\begin{array}{c}T_{1,1}+T_{4,1} \\ \vdots \\ T_{1, N}+T_{4, N}\end{array}\right]=\left[\begin{array}{cc}T_{2,1}+T_{3,1} & -2 \\ \vdots & \vdots \\ T_{2, N}+T_{3, N} & -2\end{array}\right]\left[\begin{array}{c}\theta_{1} \\ \theta_{0}\end{array}\right]+\left[\begin{array}{c}Y_{1}-X_{1} \\ \vdots \\ Y_{N}-X_{N}\end{array}\right]$

When $X_{i}$ and $Y_{i}$ are i.i.d. and follow the exponential distribution with rate parameter $\lambda$, it is easy to see that
$Z_{i} \triangleq Y_{i}-X_{i}$ follows Lapacian distribution with location parameter 0 and scale parameter $1 / \lambda$, which can be represented as $Z_{i} \sim \operatorname{Laplace}(0,1 / \lambda)$. Denote $T_{S, i}=T_{1, i}+T_{4, i}$ and $T_{P, i}=T_{2, i}+T_{3, i}$, the likelihood function can be written as

$$
\begin{aligned}
& \ln f\left(\left\{T_{S, i}, T_{P, i}\right\}_{i=1}^{N} ; \theta_{1}, \theta_{0}\right) \\
& =N \ln \frac{\lambda}{2}-\lambda \cdot \sum_{i=1}^{N}\left|T_{S, i}-\theta_{1} T_{P, i}+2 \theta_{0}\right| .
\end{aligned}
$$

Therefore, the MLE becomes minimizing the second term on the RHS of (5)

$$
\left[\theta_{1}^{*}, \theta_{0}^{*}\right]=\underset{\theta_{1}, \theta_{0}}{\arg \min } \sum_{i=1}^{N}\left|T_{S, i}-\theta_{1} T_{P, i}+2 \theta_{0}\right|,
$$

which is an unconstrained $L_{1}$-norm minimization problem. Denote the objective function as $F\left(\theta_{1}, \theta_{0}\right)$, we observe that if $\theta_{1}$ is given, $F\left(\theta_{0}\right)=2 \sum_{i=1}^{N}\left|\theta_{0}-0.5\left(\theta_{1} T_{P, i}-T_{S, i}\right)\right|$, and the MLE of $\theta_{0}$ is the median value of the sequence $\left\{0.5\left(\theta_{1} T_{P, i}-T_{S, i}\right)\right\}_{i=1}^{N}$ [8]. On the other hand, if $\theta_{0}$ is given, the MLE of $\theta_{1}$ is the optimal solution of the following problem

$$
\underset{\theta_{1}}{\arg \min } \sum_{i=1}^{N} T_{P, i}\left|\theta_{1}-\frac{T_{S, i}+2 \theta_{0}}{T_{P, i}}\right| .
$$

Treating $\left(T_{S, i}+2 \theta_{0}\right) / T_{P, i}$ as the location of the $i^{t h}$ particle with weight $T_{P, i}$ on a horizontal line, it can be proved that the MLE of $\theta_{1}$ is the so-called weighted median value of the data set $\left\{\left[T_{P, i},\left(T_{S, i}+2 \theta_{0}\right) / T_{P, i}\right]\right\}_{i=1}^{N}$ [9]. The procedure for finding the weighted median value is given as follow.

1) Sort the location sequence $\rho_{i} \triangleq\left(T_{S, i}+2 \theta_{0}\right) / T_{P, i}$ in ascending order, such that $\rho_{[1]}<\cdots<\rho_{[i]}<\cdots<\rho_{[N]}$, where $\left\{\rho_{[i]}\right\}_{i=1}^{N}$ is the order statistics of $\left\{\rho_{i}\right\}_{i=1}^{N}$ (that is, $[i]$ is the index of the $i^{t h}$ smallest element among $\left.\left\{\rho_{i}\right\}_{i=1}^{N}\right)$;

2) Find the smallest value of $K$ such that $\sum_{i=[1]}^{[K]} T_{P, i} \geq$ $0.5 \sum_{i=1}^{N} T_{P, i}$, and denote the solution as $K^{*}$;

3) The weighted median of the data set $\left\{\left[T_{P, i},\left(T_{S, i}+\right.\right.\right.$ $\left.\left.\left.2 \theta_{0}\right) / T_{P, i}\right]\right\}_{i=1}^{N}$ is $\rho_{\left[K^{*}\right]}=\left(T_{S,\left[K^{*}\right]}+2 \theta_{0}\right) / T_{P,\left[K^{*}\right]}$.

Based on the above observations, an iterative algorithm is proposed to find the optimal solution. First, the Least Squares (LS) solution is calculated as

$$
\left[\theta_{1}^{0}, \theta_{0}^{0}\right]=\left[\begin{array}{cc}
T_{P, 1} & -2 \\
\vdots & \vdots \\
T_{P, N} & -2
\end{array}\right]^{\dagger} \cdot\left[\begin{array}{c}
T_{S, 1} \\
\vdots \\
T_{S, N}
\end{array}\right]
$$

where $[\mathbf{A}]^{\dagger}$ indicates the pseudo-inverse operation of matrix A. Using the LS solution as the initial estimates of $\theta_{1}$ and $\theta_{0}$, the proposed iterative algorithm repeats the following operation until convergence.

1) Estimate $\theta_{1}^{k}$ as the weighted median value of the sequence $\left\{\left[T_{P, i},\left(T_{S, i}+2 \theta_{0}^{k-1}\right) / T_{P, i}\right]\right\}_{i=1}^{N}$

2) Estimate $\theta_{0}^{k}$ as the median value of the sequence $0.5\left\{\theta_{1}^{k} T_{P, i}-T_{S, i}\right\}_{i=1}^{N}$.

After obtaining the MLE of $\theta_{1}$ and $\theta_{0}$, we have $\beta_{1}^{*}=1 / \theta_{1}^{*}$ and $\beta_{0}^{*}=\theta_{0}^{*} / \theta_{1}^{*}$. 
Since both the weighted median and the median operations find the MLE of the corresponding parameter, we have

$$
F\left(\theta_{1}^{k-1}, \theta_{0}^{k-1}\right) \geq F\left(\theta_{1}^{k}, \theta_{0}^{k-1}\right) \geq F\left(\theta_{1}^{k}, \theta_{0}^{k}\right) .
$$

Moreover, the objective function $F\left(\theta_{1}, \theta_{0}\right)$ in (5) is convex, and there exists no local minimums. Therefore, the proposed algorithm will finally converge to the global optimal solution. And simulation results show that the algorithm usually converges within 10 iterations.

\section{Approximate Cramer-Rao Lower Bound (CRLB)}

To find CRLB, we need to take derivative of the likelihood function in (5) with respect to the unknown parameters. But the likelihood function (5) is non-differentiable at $T_{S, i}-$ $\theta_{1} T_{P, i}+2 \theta_{0}=0$. Nevertheless, (5) can be approximated with a function that is differentiable. Here we use the following approximation

$$
|t| \approx \frac{1}{r} \ln [\cosh (r t)],
$$

where $r$ is a user-defined parameter and can be used to control the accuracy of the approximation. For example, when $r=$ 200 , the function (10) gives nearly perfect approximation to the absolute value function, as shown in Fig 2.

Since $Z_{i}=T_{S, i}-\frac{1}{\beta_{1}} T_{P, i}+\frac{2 \beta_{0}}{\beta_{1}}$ and follows Laplacian distribution, i.e., $Z_{i} \sim \operatorname{Laplace}(0,1 / \lambda)$, the approximated likelihood function of (5) is given by

$$
\begin{aligned}
& \ln f\left(\left\{T_{P, i}, T_{S, i}\right\}_{i=1}^{N} ; \beta_{1}, \beta_{0}\right) \\
& \approx N \ln \frac{\lambda}{2}-\lambda \sum_{i=1}^{N} \frac{1}{r} \ln \left\{\cosh \left[r\left(T_{S, i}-\frac{1}{\beta_{1}} T_{P, i}+\frac{2 \beta_{0}}{\beta_{1}}\right)\right]\right\} .
\end{aligned}
$$

Taking second derivative of the log-likelihood function (11) with respect to $\beta_{1}$ and $\beta_{0}$, we have

$$
\begin{aligned}
\frac{\partial^{2} \ln f}{\partial^{2} \beta_{1}}= & -\lambda \sum\left(T_{P, i}-2 \beta_{0}\right)\left[-2 \beta_{1}^{-3} \tanh \left(r Z_{i}\right)\right. \\
& \left.+r \beta_{1}^{-4}\left(T_{P, i}-\beta_{0}\right) \operatorname{sech}^{2}\left(r Z_{i}\right)\right] \\
\frac{\partial^{2} \ln f}{\partial^{2} \beta_{0}}= & -\lambda \sum\left[4 r \beta_{1}^{-2} \operatorname{sech}^{2}\left(r Z_{i}\right)\right] \\
\frac{\partial^{2} \ln f}{\partial \beta_{1} \partial \beta_{0}}= & -\lambda \sum\left[-2 \beta_{1}^{-2} \tanh \left(r Z_{i}\right)\right. \\
& \left.+2 r \beta_{1}^{-3}\left(T_{P, i}-2 \beta_{0}\right) \operatorname{sech}^{2}\left(r Z_{i}\right)\right] .
\end{aligned}
$$

To calculate CRLB, we further need to take expectation of (12) - (14) with respect to $Z_{i}$. Based on the fact that $\int_{0}^{+\infty} \tanh (x) e^{-\mu x} \mathrm{~d} x=-\int_{-\infty}^{0} \tanh (x) e^{\mu x} \mathrm{~d} x$, we have

$$
\int_{-\infty}^{+\infty} \tanh \left(r Z_{i}\right) \frac{\lambda}{2} e^{-\lambda Z_{i}} \mathrm{~d} Z_{i}=0 .
$$

Furthermore, we also have

$$
\begin{aligned}
& \int_{-\infty}^{+\infty} \operatorname{sech}^{2}\left(r Z_{i}\right) \frac{\lambda}{2} e^{-\lambda Z_{i}} \mathrm{~d} Z_{i} \\
& =\frac{\lambda}{r} \underbrace{\left\{\frac{\lambda}{2 r}\left[\psi\left(\frac{\lambda+2 r}{4 r}\right)-\psi\left(\frac{\lambda}{4 r}\right)\right]-1\right\}}_{\triangleq V},
\end{aligned}
$$

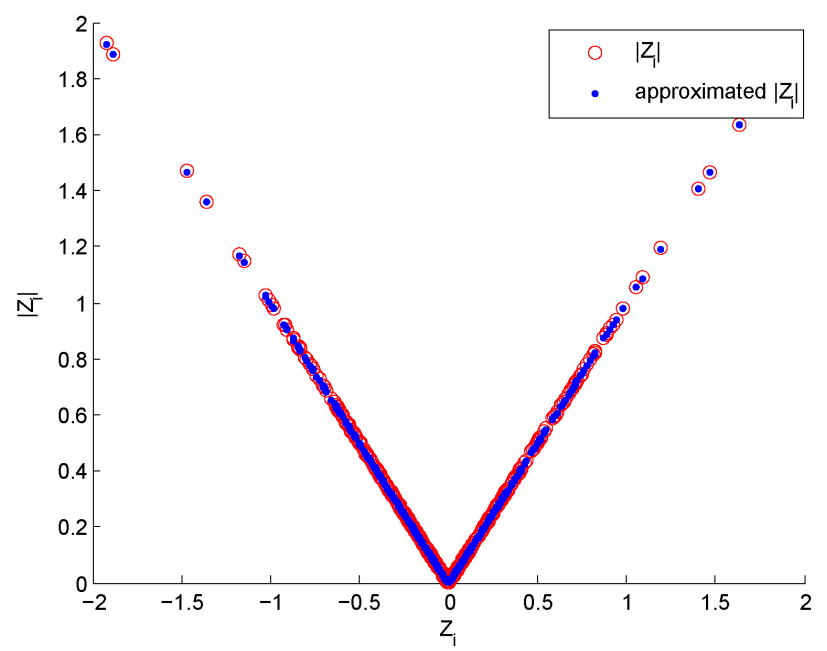

Fig. 2. Approximation of the Laplacian random variable.

because $\int_{0}^{+\infty} \operatorname{sech}^{2}(x) e^{-\mu x} \mathrm{~d} x=\int_{-\infty}^{0} \operatorname{sech}^{2}(x) e^{\mu x} \mathrm{~d} x=$ $\frac{\mu}{2}\left[\psi\left(\frac{\mu+2}{4}\right)-\psi\left(\frac{\mu}{4}\right)\right]-1$, where $\psi(x)$ is a special function named Euler psi function and its precise definition can be found in [10, pp.892-896].

Using (15) and (16), it is easy to calculate the expectation of (12) - (14) with respect to $Z_{i}$, and the Fisher information matrix is

$$
\begin{aligned}
\operatorname{FIM}\left(\beta_{1}, \beta_{0}\right) & =\left[\begin{array}{cc}
-\mathbf{E} \frac{\partial^{2} \ln f}{\partial \beta_{1}^{2}} & -\mathbf{E} \frac{\partial^{2} \ln f}{\partial \beta_{1} \partial \beta_{0}} \\
-\mathbf{E} \frac{\partial^{2} \ln f}{\partial \beta_{0} \partial \beta_{1}} & -\mathbf{E} \frac{\partial^{2} \ln f}{\partial \beta_{0}^{2}}
\end{array}\right] \\
& =\frac{\lambda^{2}}{\beta_{1}^{4}}\left[\begin{array}{ll}
\mathcal{A} & \mathcal{B} \\
\mathcal{B} & \mathcal{C}
\end{array}\right],
\end{aligned}
$$

where $\mathcal{A} \triangleq \sum_{i=1}^{N} V\left(T_{P, i}-2 \beta_{0}\right)^{2}, \mathcal{B} \triangleq 2 \beta_{1} \sum_{i=1}^{N} V\left(T_{P, i}-\right.$ $\left.2 \beta_{0}\right)$ and $\mathcal{C} \triangleq 4 \beta_{1}^{2} N V$. By inverting the matrix (17), it can be shown that the CRLB for each parameter is

$$
\begin{aligned}
& \operatorname{CRLB}\left(\beta_{1}\right)=\frac{\beta_{1}^{4} \mathcal{C}}{\lambda^{2}\left(\mathcal{A C}-\mathcal{B}^{2}\right)}, \\
& \operatorname{CRLB}\left(\beta_{0}\right)=\frac{\beta_{1}^{4} \mathcal{A}}{\lambda^{2}\left(\mathcal{A C}-\mathcal{B}^{2}\right)} .
\end{aligned}
$$

\section{Simulation RESUlts AND DISCUSSIONS}

As mentioned in Section I, Noh et al. [6] proposed an algorithm EMLLE for joint estimation of the clock offset and clock skew. Here, simulation results are presented to compare the performances of the EMLLE and the proposed estimator. The parameters used in the simulation are $\lambda=1, d=2$, $\beta_{1}=1.003$ and $\beta_{0}=-10$. Each point in the figures is an average of 10000 simulation runs.

Fig. 3 shows the mean squared error (MSE) for estimation of the clock skew $\beta_{1}$ as a function of the number of round of message exchange $N$. As shown in the figure, the proposed estimator performs much better than EMLLE. The performance of the proposed estimator deviates slightly from the CRLB, which is due to the approximation, and similar behavior is also found in [11]. However, the approximate CRLB can still predict the trend of the performance of the proposed estimator 


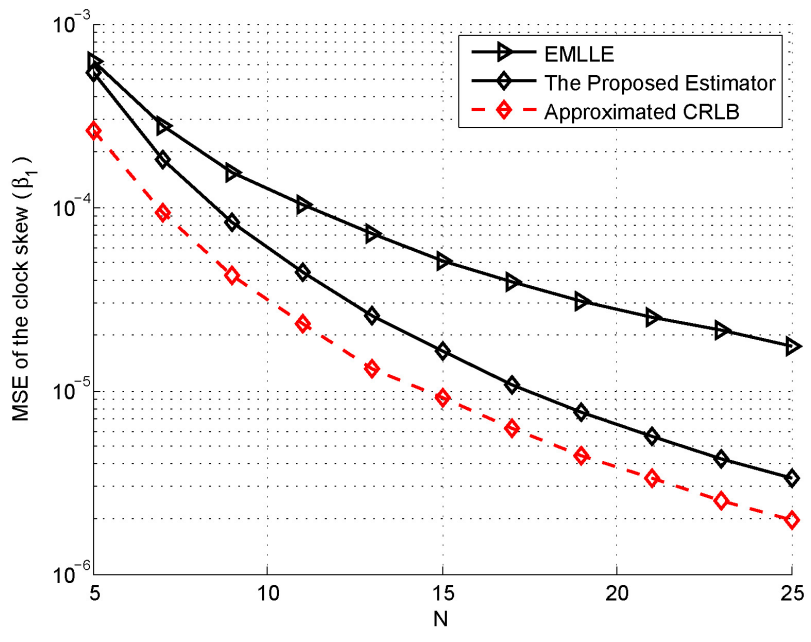

Fig. 3. MSE of estimated clock skew $\hat{\beta_{1}}$ with respect to the number of rounds of message exchange $N$.

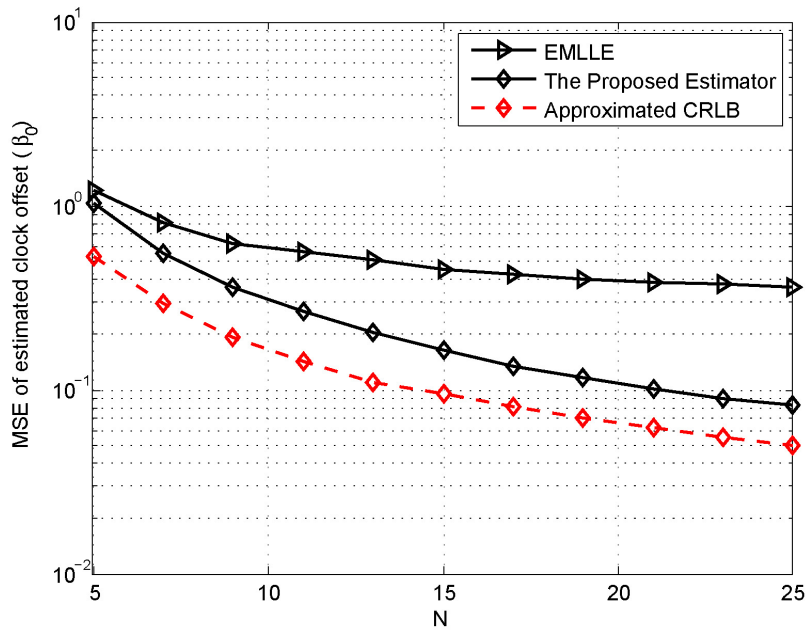

Fig. 4. MSE of estimated clock offset $\hat{\beta}_{0}$ with respect to the number of rounds of message exchange $N$.

and serve as the lowest limit. Fig. 4 shows the corresponding results for the clock offset $\beta_{0}$. It can be seen from the figure, the same conclusions as in Fig. 3 can be drawn.

\section{CONCLUSIONS}

Clock synchronization for WSN in the presence of exponential delay was discussed based on the two-way message exchange mechanism. The MLE for joint estimation of clock skew and clock offset was derived by treating the unknown fixed delay as a nuisance parameter. The CRLB was also derived by approximating the original non-differentiable probability density function with a highly accurate differentiable function. Although the performance of the proposed estimator deviates slightly from CRLB, which seems to be a common result due to the mild approximations [11], the CRLB predicts the trend of the performance very well and gives the lowest limit. The proposed estimator was also compared to an existing algorithm, and simulation results indicate that the proposed estimator gives improved performance compared to the EMLLE.

\section{REFERENCES}

[1] I. F. Akyildiz, W. Su, Y. Sankarasubramaniam, E. Cayirci, "Wireless sensor networks: a survey", Cumputer Networks, vol. 38, no. 4, pp. 393-422, Mar. 2002.

[2] N. Bulusu and S. Jha, Wireless Sensor Networks: A Systems Perspective. Artech House, 2005.

[3] H. S. Abedel-Ghaffar, "Analysis of synchronization algorithms with time-out control over networks with experimentally symmetric delays", IEEE Trans. On Communications. vol. 50, no. 10, pp. 1652-1661. Oct. 2002

[4] H. Kopetz and W. Ochsenreiter, "Clock synchronization in distributed real-time systems", IEEE Trans. on Computers., vol. 36, no. 8, pp. 933940, Aug. 1987.

[5] D. R. Jeske, "On the maximum likelihood estimation of clock offset", IEEE Trans. on Communication., vol.53 pp. 53-54, Jan. 2005.

[6] K. L. Noh, Q. M. Chaudhari, E. Serpedin and B. W. Suter, "Novel clock phase offset and skew estimation using two-way timing message exchanges for wireless sensor networks", IEEE Trans. on Communications. vol. 55, no. 4, pp. 766-777, April 2007.

[7] M. Leng and Y. C. Wu, "On clock synchronization algorithms for Wireless Sensor Networks with unknown delay", to appear in IEEE Trans. on Vehicular Technology.

[8] Junpei Sekino, "n-Ellipse and the minimum distance sum problem", The American Mathematical Monthly, vol. 106, No. 3, pp. 193-202, Mar., 1999.

[9] Chaya Gurwitz, "Weighted median algorithms for $L_{1}$ approximation", BIT Numerical Mathematics, 301-310. June 1990.

[10] I. S. Gradshteyn and I. M. Ryzhik, Table of integrals, series, and products. The sixth edition, Academic press. July 2000.

[11] Esa Ollila et al., "Compact Cramer-Rao Bound expression for independent component analysis", IEEE Trans. on Signal Processing, vol.56, No.4, pp. 1421-1428, April 2008. 